\title{
Protein Arginine Methyltransferase 1 Methylates Smurf2
}

\author{
Boksik Cha, ${ }^{1,2}$, Yaerin Park, ${ }^{1,2}$, Byul Nim Hwang', So-young Kim ${ }^{1}$, and Eek-hoon Jho, *
}

\begin{abstract}
Smurf2, a member of the HECT domain E3 ligase family, is well known for its role as a negative regulator of TGF- $\beta$ signaling by targeting Smads and TGF- $\beta$ receptor. However, the regulatory mechanism of Smurf2 has not been elucidated. Arginine methylation is a type of post-translational modification that produces monomethylated or dimethylated arginine residues. In this report, we demonstrated methylation of Smurf2 by PRMT1. In vitro methylation assay showed that Smurf2, not Smurf1, was methylated by PRMT1. Among the type I PRMT family, only PRMT1 showed activity for Smurf2. Transiently expressed Smurf2 was methylated by PRMT1, indicating Smurf2 is a novel substrate of PRMT1. Using deletion constructs, methylation sites were shown to be located within amino acid region 224-298 of Smurf2. In vitro methylation assay following point mutation of putative methylation sites confirmed the presence of Arg232, Arg234, Arg237, and Arg239. Knockdown of PRMT1 resulted in increased Smurf2 expression as well as inhibition of TGF- $\beta$-mediated reporter activity. Although it is unclear whether or not increased Smurf2 expression can be directly attributed to lack of methylation of arginine residues, our results suggest that methylation by PRMT1 may regulate Smurf 2 stability and control TGF- $\beta$ signaling.
\end{abstract}

\section{INTRODUCTION}

Protein post-translational modifications such as phosphorylation, acetylation, glycosylation, ubiquitination, and methylation, or reversal of such modifications, can provide structural and functional diversity among proteins. Ubiquitination is a type of post-translational modification that controls cell division, differentiation, and signal transduction through protein stability, protein trafficking, and protein-protein interactions (Hershko and Ciechanover, 1998; Mukhopadhyay and Riezman, 2007). Ubiquitination is performed sequentially by three types of enzymes. E1 ubiquitin-activating enzyme forms a thioester bond between the active cysteine and $\mathrm{C}$-terminal glycine of ubiquitin

\footnotetext{
1Department of Life Science, University of Seoul, Seoul 130-743, Korea, ${ }^{2}$ These authors contributed equally to this work.

*Correspondence: ej70@uos.ac.kr
}

Received 30 April, 2015; revised 13 May, 2015; accepted 14 May, 2015; published online 1 July, 2015

Keywords: methylation, PRMT1, signal transduction, Smurf2, TGF- $\beta$ in an ATP-dependent manner. Activated ubiquitin is then transferred to the active cysteine of E2 ubiquitin-conjugating enzyme. Finally, E3 ubiquitin ligase transfers E2-conjugated ubiquitin to lysine residues of specific substrates (Pickart, 2001). E3 ubiquitin ligases are divided into two types based on catalytic domain, HECT (homologous to E6-AP carboxyl terminus) domain and RING (really interesting new gene) finger domain (Deshaies and Joazeiro, 2009; Rotin and Kumar, 2009).

Smad ubiquitination regulatory factor 2 (Smurf2) was initially identified as Smurf1-related HECT domain E3 ligase (Zhang et al., 2001) and is a negative regulator of TGF- $\beta$ signaling by controlling steady state levels of Smads and type I receptor (Dupont et al., 2012; Izzi and Attisano, 2004; Lonn et al., 2009). In addition, it has been reported that Smurf2 controls levels of non TGF- $\beta$ signaling components. For example, Smad7associated $\beta$-catenin, a positive regulator of Wnt signaling, was shown to be degraded by Smurf2 in a Smad7 transgenic mice model (Han et al., 2006). Moreover, abnormal overexpression of Smurf2 has been reported in several types of human cancers (Fukuchi et al., 2002; Jin et al., 2009), whereas a knockout mice study showed that loss of Smurf2 promotes tumorigenesis (Blank et al., 2012). Although there are many reports that Smurf2 regulates a variety of cellular processes by targeting diverse substrates, it is still unknown how Smurf2 activity is controlled in a pathophysiological context.

Arginine methylation, a type of post-translation modification, involves the addition of monomethyl or dimethyl groups to arginine residues, resulting in structural protein changes (Cha and Jho, 2012). Protein arginine methyltransferases (PRMTs) are responsible for such a modification (Bedford, 2007). Among the PRMT family, PRMT1 covers greater than $85 \%$ arginine methylation activity (Tang et al., 2000). PRMT1 knockout mice die at E6.5, implying that PRMT1 has non-redundant and essential roles in embryonic development (Pawlak et al., 2000). Moreover, PRMT1 plays a role in the regulation of various biological functions such as transcriptional activation, signal transduction, protein trafficking, DNA repair, and protein-protein interactions as well as dysregulation of human diseases (Bedford and Clarke, 2009; McBride and Silver, 2001).

Until now, there has been no report of Smurf2 modified by post-translational modifications. In this report, we provide evidence that PRMT1 is capable of methylating Smurf2 in vitro. Although we could not determine the significance of Smurf2 methylation by PRMT1 in a physiological setting, our data suggest that methylation of Smurf2 by PRMT1 may regulate stability of Smurf2 and control TGF- $\beta$ signaling. 


\section{MATERIALS AND METHODS}

\section{Plasmids and siRNAs}

Human Smurf2 cDNA was obtained using total RNA from a human cell line and inserted into pGEX vectors as described previously (Kim and Jho, 2010). Mouse PRMT1 and PRMT4 cDNAs were obtained from the total RNA of mouse embryos (Cha et al., 2011). pGEX-PRMT3, pGEX-PRMT6, and pGEXGAR were kindly provided from Drs. Herschman (UCLA, USA) and Bedford (The University of Texas, USA). GST-Smurf2 deletion constructs were generated using PCR and a general molecular cloning technique. Smurf2 constructs were used for sitedirected mutagenesis at R232, R234, R237, and R239 of Smurf2. siRNA oligonucleotides targeting GFP were used as a negative control (Tiscornia et al., 2003), and siRNA targeting PRMT1 was purchased from ST Pharm (Yamagata et al., 2008).

\section{Cell culture and transient transfection}

HEK293T human embryonic kidney cells were grown in Dulbecco's modified Eagle's medium (DMEM, Invitrogen) supplemented with $10 \%$ fetal bovine serum (FBS, Cambrex Bio Science Walkersville, Inc.) and $1 \mathrm{X}$ antibiotic-antimycotic (Invitrogen) and maintained at $37^{\circ} \mathrm{C}$ in a humidified $5 \% \mathrm{CO}_{2}$ incubator. Transient transfection of plasmids into HEK293T was performed by the calcium phosphate precipitation method or by using WelFect-EXTM Plus transfection reagent (WelGENE). siRNA was transfected with lipofectamine RNAi max (Invitrogen).

\section{Immunoprecipitation, immunoblotting, and antibodies}

Cells were lysed in lysis buffer (20 mM Tris- $\mathrm{HCl}(\mathrm{pH} 7.5), 150$ $\mathrm{mM} \mathrm{NaCl}, 1.0 \%$ Triton X-100, $20 \mathrm{mM} \mathrm{NaF}, 2$ mM EDTA, 2 mM Na-orthovanadate, $1 \mathrm{mM} \mathrm{PMSF}$, and $5 \mu \mathrm{g} / \mathrm{ml}$ leupeptin A), after which the concentrations of proteins were measured using Bradford reagent (Bio-Rad). For immunoprecipitation, 600-800 $\mu \mathrm{g}$ of cell lysates was incubated with proper antibody in lysis buffer overnight at $4^{\circ} \mathrm{C}$ with constant rotation. For in vitro binding assays, GST-fused proteins were expressed in BL21 bacterial cells and purified using glutathione-agarose beads (GE Healthcare). Proteins harboring $\left[S^{35}\right]$-labeled methionine (Met) were generated by an in vitro translation system (Invitrogen). The immunoprecipitates were analyzed by SDS-PAGE and immunoblotting. Mouse monoclonal anti-PRMT1 antibody (clone 171, Sigma), mouse monoclonal anti-mono/dimethyl arginine antibody (7E6, AbCam), anti-Flag monoclonal antibody (M2, Sigma), and anti-Smurf2 (D8B8, Cell Signaling) were used. Peroxidase-conjugated goat anti-mouse and anti-rabbit secondary antibodies (Santa Cruz biotechnology) were used, and proteins were detected by using enhanced chemiluminescence reagent (ELPIS or Milipore).

\section{In vitro methylation assay}

In vitro methylation assays were performed as described previously (Bedford et al., 2000). In brief, GST-PRMT1 (mouse), PRMT3 (mouse), PRMT4 (mouse), PRMT6 (Rat), and various GST-Smurf2 constructs were purified using GlutathioneAgarose 4B (Peptron). Various GST-Smurf2 constructs $(0.5 \mu \mathrm{g})$ were incubated with indicated GST-PRMTs $(0.2-1.0 \mu \mathrm{g})$ in the presence of $2 \mu \mathrm{l}$ of S-adenosyl-L-[methyl- $\left.{ }^{3} \mathrm{H}\right]$ methionine ([ $\left.{ }^{3} \mathrm{H}\right]$ AdoMet; $83.3 \mathrm{ci} / \mathrm{mmol}$, Perkin Elmer) for the indicated time at $30^{\circ} \mathrm{C}$ in $20 \mu$ of methylation reaction buffer $(15 \mathrm{mM}$ Tris- $\mathrm{HCl}$ (pH.7.5), $25 \mathrm{mM} \mathrm{NaCl} 10 \mathrm{mM}$ EDTA). Methylation reactions were stopped by the addition of $4 \mathrm{X}$ SDS sample buffer, followed by heating $100^{\circ} \mathrm{C}$ for $10 \mathrm{~min}$. Samples were separated on SDS-PAGE and stained with $0.05 \%$ Coomassie Brilliant Blue. After destaining, gels were soaked in EN³ HANCE (PerkinElmer) according to the manufacturer's instructions and visualized by fluorography after exposure for 1 day to 1 week at $-80^{\circ} \mathrm{C}$.

For in vitro binding assay, bacterially expressed GST-Smurf2 were purified using glutathione-agarose beads (GE Healthcare) and incubated with $\left[\mathrm{S}^{35}\right]$-labelled Myc-PRMT1, which was generated by an in vitro translation system (Invitrogen). After immunoprecipitation, the samples were analyzed by SDSPAGE, followed by immunoblotting.

\section{Luciferase assay}

HEK 293 cells were seeded in 12-well plates for 1 day before transfection. To check TGF- $\beta$ signaling, Smad3/4-specific $(\mathrm{CAGA})_{12}$-MLP-luciferase reporter plasmid, SBE4-luciferase reporter plasmid containing four Smad3,4-binding sequences, or TGF- $\beta$-inducible construct p3TP-Lux was co-transfected with thymidine kinase promoter-driven Renilla luciferase ( $\mathrm{pRL}-\mathrm{TK}$ ) and the indicated plasmids. After 4-6 $\mathrm{h}$ of transfection, media were exchanged with pre-warmed fresh media containing 200 nM TGF- $\beta$ ligands. The next day, cells were lysed and luciferase activity measured using a dual-luciferase reporter assay system (Promega) according to the manufacturer's instructions. Luciferase activity was measured by a GLOMAX 20/20 luminometer (Promega). Transfection efficiency was normalized to thymidine kinase promoter-driven Renilla luciferase (pRL-TK) activity as the internal control.

\section{RESULTS}

\section{Methylation of Smurf2 by PRMT1}

To determine whether or not Smurf2 is a substrate of PRMT1, we examined methylation of GST-Smurf2 by GST-PRMT1 in vitro. As seen in Fig. 1A, only wild-type GST-PRMT1 methylated GST-Smurf2. Methylation-dead (MD) GST-PRMT1, which lacks methylation activity (McBride et al., 2000), showed no such activity. Furthermore, methylation of Smurf2 was elevated by PRMT1 in a time- and dose-dependent manner (Figs. 1B and $1 \mathrm{C}$ ). To confirm that PRMT1 can methylate Smurf2 in vivo, we transfected HA epitope-tagged Smurf2 (HA-Smurf2) with or without Flag epitope-tagged PRMT1 (Flag-PRMT1) or FlagMD-PRMT1 into HEK293T cells. Methylated Smurf2 immunoprecipitated using HA antibody was detected by anti-methylarginine (Methyl-R) antibody, which specifically recognizes methylated arginine residues (Goulet et al., 2007) (Fig. 1D). Since Smurf2 was identified as a Smurf1-related member of HECT domain E3 ligase and has almost $80 \%$ sequence identity with Smurf1 (Zhang et al., 2001), we assumed that Smurf1 could be methylated by PRMT1. However, as seen Fig. 1E, only Smurf2 was methylated by PRMT1. In addition, among the type I PRMT family, including PRMT1, PRMT3, PRMT4/Carm1, and PRMT6 (Bedford and Clarke, 2009), only PRMT1 specifically methylated Smurf2 (Fig. 1F).

\section{Smurf2 interacts with PRMT1 in vitro and in vivo}

Since Smurf2 was methylated by PRMT1 in vivo and in vitro (Fig. 1), we tested whether or not Smurf2 interacts with PRMT1. Immunoprecipitation analysis of HEK-293T cells transfected with HA-tagged Smurf2 and Flag-tagged PRMT1 showed that exogenously expressed Smurf2 interacted with PRMT1 (Fig. 2A). To examine whether or not PRMT1 directly binds to Smurf2, in vitro binding assay was performed. In vitro translated Myc-PRMT1 labeled with ${ }^{35} \mathrm{~S}$-methionine was incubated with bacterially purified GST or GST-Smurf2. As shown in Fig. 2B, 


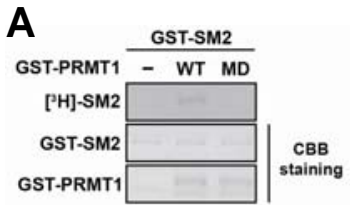

D

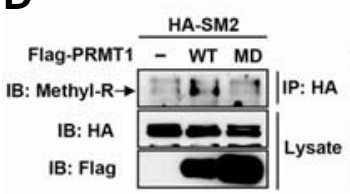

B

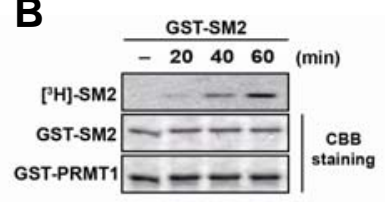

E

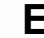

$F$
C

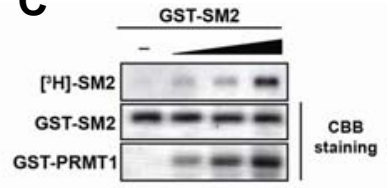

E GST-SM1 GST-SM2

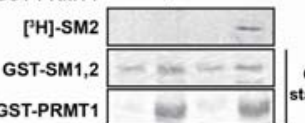

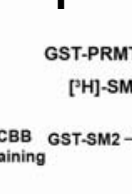
H]-SM
GST-SM2

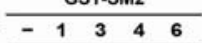

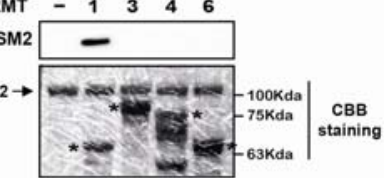

Fig. 1. Methylation of Smurf2 by PRMT1. (A) In vitro methylation assays. Purified GST-Smurf2 (SM2) was incubated with purified wild-type (WT) or methylation-dead (MD) GSTPRMT1 in the presence of [methyl- ${ }^{3} \mathrm{H}$ ] adenosylmethionine (SAM). Reaction products were analyzed by SDSPAGE, followed by fluorography (upper panel). Total amounts of GSTSmurf2 and GST-PRMT1 were determined by Coomassie brilliant blue (CBB) staining. $(\mathrm{B}, \mathrm{C})$ In vitro methylation of Smurf2 in a time-dependent manner or PRMT1 dose-dependent manner. (D) Ectopic expression of PRMT1 enhances methylation of Smurf2. HA-Smurf2 was transfected alone or co-transfected with Flag-PRMT1 or Flag-MD-PRMT1 into HEK293T cells. Cell lysates were immunoprecipitated with anti-HA antibody and then immunoblotted with anti-mono/dimethyl-arginine (Methyl-R) antibody. (E) Smurf1 is not methylated by PRMT1. GST-PRMT1 was incubated with GST-Smurf1 or GST-Smurf2 in the presence of $\left.{ }^{3} \mathrm{H}\right] \mathrm{SAM}$. Methylation was visualized by fluorography (upper panel). (F) Only PRMT1 methylates Smurf2. GST-Smurf2 was incubated with GST-PRMT1 or PRMT3, PRMT4, and PRMT6 in the presence of [ ${ }^{3} \mathrm{H}$ ] SAM. Arrow indicates Smurf2, and asterisks indicate PRMT family.
$\boldsymbol{A}$

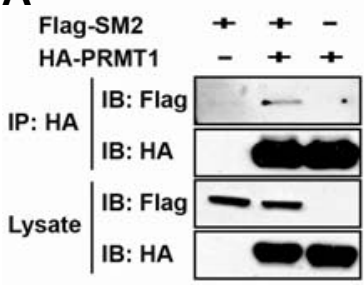

C

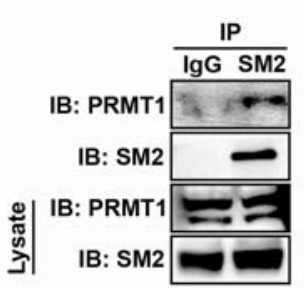

B

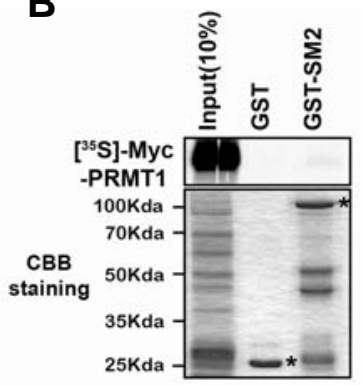

D

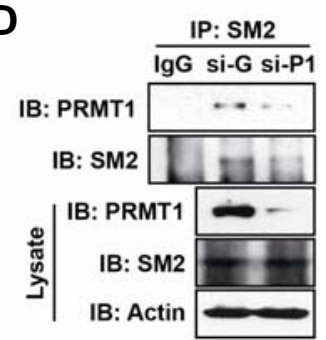

Fig. 2. Smurf2 interacts with PRMT1 in vitro and in vivo. (A) Coimmunoprecipitation assay of ectopically expressed Smurf2 and PRMT1. HA-tagged Smurf2 and Flag-tagged PRMT1 were transfected alone or together into HEK-293T cells. After $24 \mathrm{~h}$, cells were subjected to immunoprecipitation, followed by immunoblotting with antiHA or anti-Flag antibodies as indicated. (B) Direct interaction between Smurf2 and PRMT1. Myc-tagged PRMT1, in vitro labeled with [ $\left.{ }^{35} \mathrm{~S}\right]-$ methionine, was incubated with glutathione-sepharose bead-bound GST-Smurf2 fusion protein. GST-Smurf2 was pulled down by beads, after which interaction with PRMT1 was visualized by autoradiography. GST and GST-Smurf2 are shown by CBB staining. Asterisks indicate GST or GST-Smurf2. (C) Smurf2 endogenously interacts with PRMT1. HEK-293T cell lysates were immunoprecipitated (IP) with control rabbit lgG or rabbit anti-Smurf2 antibody, followed by immunoblotting (IB) with antibodies as indicated on the left side. (D) Knockdown of PRMT1 led to reduced interaction with endogenous Smurf2. HEK293T cells were treated with si-GFP or si-PRMT1 RNA for 72 h. Endogenous Smurf2 was immunoprecipitated with antiSmurf2 antibody, followed by immunoblotting with PRMT1 antibody.

Smurf2 directly interacted with PRMT1. Immunoprecipitation with anti-Smurf2 antibody, followed by immunoblotting with antiPRMT1 antibody using HEK-293T cell lysates showed that PRMT1 associated with Smurf2 endogenously (Fig. 2C). Knockdown of PRMT1 led to reduced interaction with endogenous Smurf2 (Fig. 2D). These results imply that Smurf2 is directly associated with PRMT1 in vivo and in vitro.

\section{Identification of methylation sites of Smurf2}

To identify the specific methylation site(s) in Smurf2, we initially constructed deletion constructs of Smurf2, as shown in Fig. 3A. Each purified GST-Smurf2 fragment was incubated with GSTPRMT1, and only deletion constructs containing amino acids 224-298 were methylated by PRMT1 (Fig. 3B). Based on these findings, we assumed that methylation sites could be located in amino acid region 224-298. PRMT1 methylates substrates containing specific arginine residues surrounded by one or more glycine residues, called a glycine- and arginine-rich (GAR) motif, or arginine residues within sequence arginineamino acid X-arginine (RXR) motifs (Boisvert et al., 2005). Therefore, we searched putative methylation sites, such as GAR and RXR motifs within amino acid region 224-298 of Smurf2. Although GAR motif was not detected within this region, two RXR motifs (232-RVR-234 and 237-RHR-239) were shown to be highly conserved in Smurf2 among different species (Fig. 3C). To determine whether or not putative methylation sites are targeted by PRMT1, arginine residues Arg232, Arg234, Arg237, and Arg239 were initially substituted by alanine, either individually or in combination. Each full-length and fragment GSTSmurf2 (224-335) mutant was used for methylation assay. As shown in Fig. 4A, single-substituted GST-Smurf2 mutants showed significantly less methylation than wild-type GSTSmurf2. Interestingly, among single point mutants, methylation states of GST-Smurf2 (R234A) and GST-Smurf2 (R239A) were diminished compared to those of GST-Smurf2 (R232A) and GST-Smurf2 (R237A), suggesting that Arg234 and Arg239 could be major methylation sites of Smurf2 or are dimethylated while Arg232 and Arg247 are mono-methylated. Consistent with this result, in vitro methylation assay using GST-Smurf2 fragments (224-335) and mutants showed similar results. Moreover, double substitution mutants GST-Smurf2-224-335 

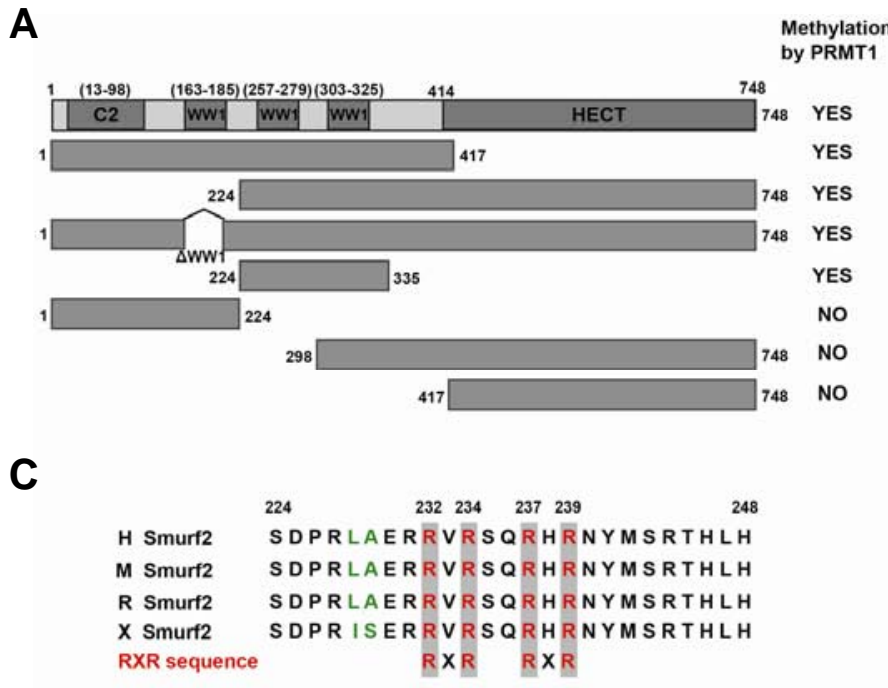

$\boldsymbol{B}$

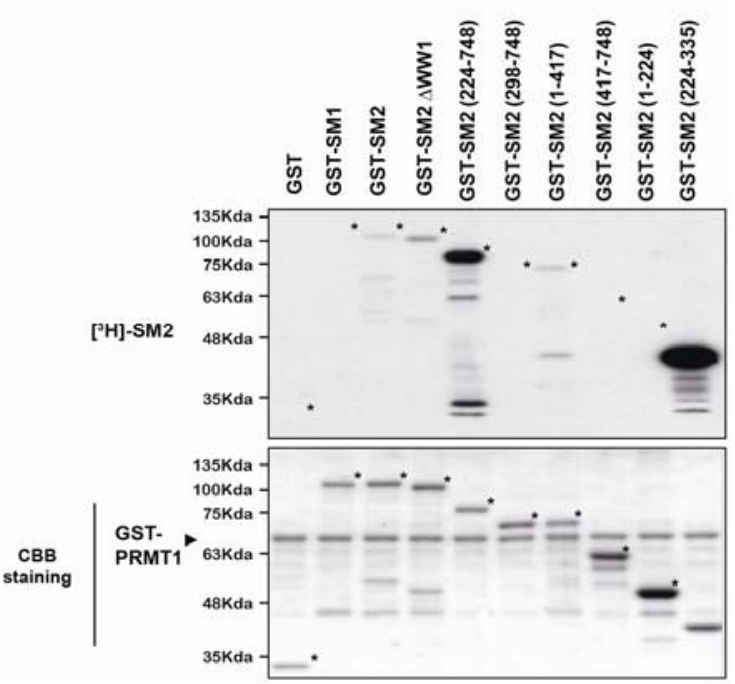

Fig. 3. Mapping of methylation domains of Smurf2 by PRMT1. (A) Schematic diagram of deletion constructs of Smurf2 and methylation results are summarized on the right side. (B) In vitro methylation assay. Purified GST fusion proteins indicated in Fig. 3A were incubated with PRMT1 in the presence of $\left[{ }^{3} \mathrm{H}\right]$ adenosylmethionine (SAM). Reaction products were analyzed by SDS-PAGE, followed by fluorography. Arrowheads indicate GST-PRMT1, and asterisks indicate GST, GST-Smurf1 (SM1), and various GST-Smurf2 (SM2) deletion constructs. (C) Conservation of putative methylation sites of human $(H)$, mouse $(M)$, rat $(R)$, and Xenopus $(X)$ Smurf2. Arg232, Arg234, Arg237, and Arg239 of Smurf2 are highly conserved among all Smurf2 proteins. Two amino acids of Xenopus, marked with green color, are different from others.

$\boldsymbol{A}$

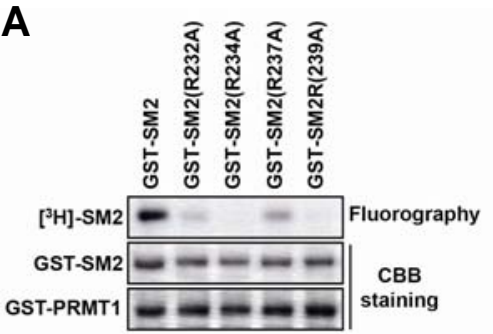

$\boldsymbol{B}$

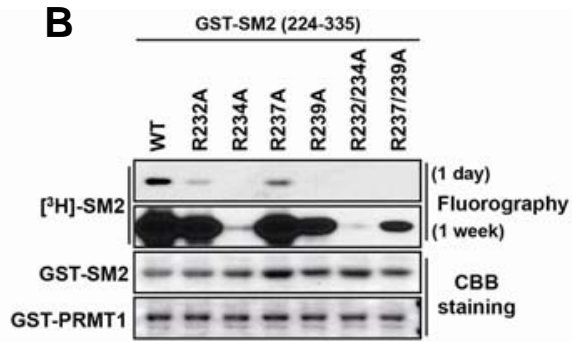

Fig. 4. PRMT1 methylates Smurf2 preferentially at Arg232, Arg234, Arg237, and Arg239. (A) In vitro methylation assays using GSTPRMT1 together with GST-Smurf2 in the presence of $\left[{ }^{3} \mathrm{H}\right] \mathrm{SAM}$. Single substitution mutants of GST-Smurf2 with arginine residues substituted by alanine were used as substrates. Total amounts of GST-Smurf2 and GST-PRMT1 were determined by CBB staining. (B) In vitro methylation assays were per-

formed with GST-Smurf2 (224-335) in the presence of $\left[{ }^{3} \mathrm{H}\right]$ SAM. Single or double substitution mutants of GST-Smurf2 (224-335) were methylated by GST-PRMT1. Reactions were exposed for 1 day or 1 week.

(R232/234A) and GST-Smurf2-224-335 (R237/239A) showed decreased methylation compared with single substitution mutants. Intriguingly, substitution of Arg234 to alanine resulted in less methylation than double substitution of Arg237 and Arg239 to alanine by PRMT1. Taken together, these data indicate the presence of four methylation sites (Arg232, Arg234, Arg237, and Arg239) in Smurf2, and PRMT1 methylation activity shows specificity for Arg234 and Arg239.

\section{Role of PRMT1-mediated methylation of Smurf2 in TGF- $\beta$ signaling}

Based on the results of PRMT1-mediated methylation of Smurf2, we next investigated biological significance of Smurf2 methylation. Smurf2 is well known for its inhibitory role in TGF- $\beta$ signaling by inducing ubiquitination/proteasome-mediated degradation of Smad proteins and TGF- $\beta$ receptors. Therefore, we tested whether or not PRMT1-mediated methylation regulates TGF- $\beta$ signaling using a Smad3/4-specific (CAGA) 12 -MLPluciferase reporter construct (Fig. 5A) (Lonn et al., 2009). TGF- $\beta$ ligand treatment increased TGF- $\beta$ signaling reporter activity, whereas ectopic expression of wild-type Smurf2 resulted in reduced activity. As expected, overexpression of catalytically inactive Smurf2 failed to reduce TGF- $\beta$-mediated reporter activity. Further, co-transfection of wild-type PRMT1 or catalytically inactive PRMT1 had no effect on the inhibitory role of Smurf2 in TGF- $\beta$ signaling. Based on this result, we could assume that PRMT1-mediated Smurf2 methylation does not regulate the inhibitory role of Smurf2 in TGF- $\beta$ signaling.

Our result which shows ectopic expression of PRMT1 had no effect on the inhibitory role of Smurf2 could be because only a small portion of Smurf2 was methylated by PRMT1. In other words, the residual non-methylated form of Smurf2 may be sufficient to inhibit TGF- $\beta$-mediated reporter activity. In order to exclude this possibility, we generated Smurf2 (R234/239A) and Smurf2 (R234/239F) mutants, which are non-methylated and methylation mimetic forms, respectively. The effect on TGF- $\beta$ signaling was checked using CAGA luciferase reporter (Fig. 5B). Although the R234/239A and R234/239F mutants were 

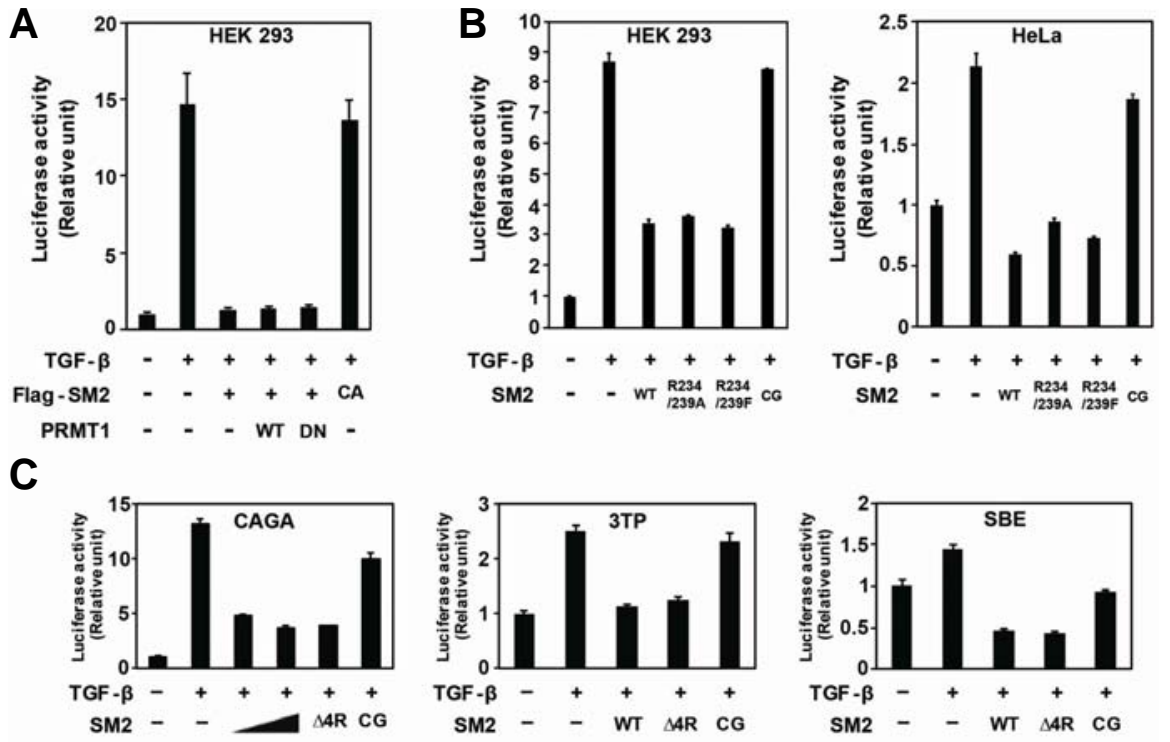

$\boldsymbol{A}$
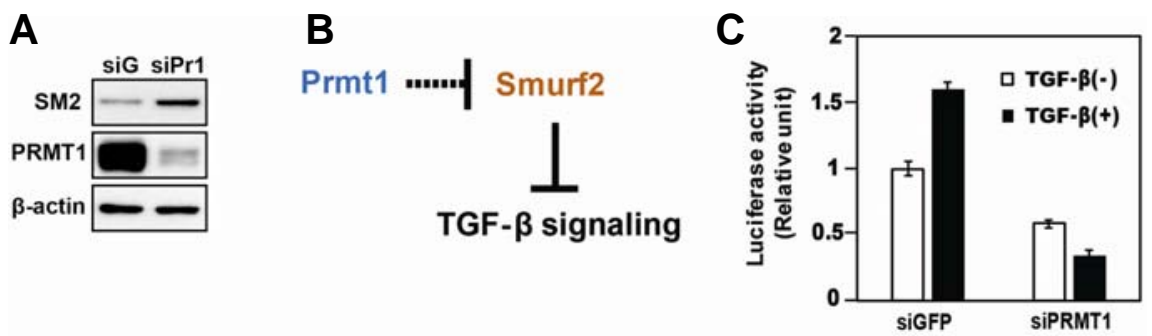

Fig. 5. PRMT1-mediated methylation has no effect on inhibitory role of Smurf2 in TGF- $\beta$ signaling. (A-C) TGF- $\beta$ signaling was measured based on Smad3/4specific CAGA12-luciferase activity (A, B). pCAGA12-luc, 3TP-luc, or SEB-luc construct was co-transfected with thymidine kinase promoter-driven Renilla luciferase (pRL-TK) and indicated plasmids (C). TGF- $\beta$ was treated overnight, and relative luciferase activity was measured. CG; catalytic inactive form, R234/239A; non-methylation mimetic form, R234/239F; methylation mimetic form, $\Delta 4 \mathrm{R}$; deletion of seven amino acids, including four arginines. Data represent average values from one representative experiment performed in triplicate. Error bars indicate standard deviations of triplicate.

Fig. 6. PRMT1-mediated methylation regulates stability of Smurf2. (A) Knockdown of PRMT1 increased level of Smurf2. Hela cells were transfected with siPRMT1 for 48-72 $\mathrm{h}$ and analyzed by immunoblotting. (B) Schematic diagram for working hypothesis. Prmt2 negatively regulates stability of Smurf2. (C) Knockdown of PRMT1 reduced TGF- $\beta$-mediated reporter activity. HeLa cells were co-transfected with pCAGA12-luc and pRL-TK after knockdown of PRMT1, and luciferase activity was measured. Data represent average values from one representative experiment performed in triplicate. Error bars indicate standard deviations of triplicate.

expected to show opposite effects on TGF- $\beta$ signaling, both mutants inhibited TGF- $\beta$ signaling similar to wild-type Smurf2 did in two different cell lines. In addition, methylation-deficient Smurf2 lacking seven amino acids, including four arginines, showed a similar inhibitory effect as wild-type Smurf2 using all three different luciferase constructs (Fig. 4C). These results suggest that PRMT1-mediated methylation at these sites is not involved in the regulation of Smurf2 activity in the context of TGF- $\beta$ signaling, at least in our overexpression experimental setting.

Interestingly, we observed that knockdown of PRMT1 increased the level of Smurf2 (Fig. 6A). We hypothesize that PRMT1 might regulate Smurf2 stability through methylation of Smurf2 (Fig. 6B). RT-PCR analysis showed that the mRNA level of Smurf2 did not change (data not shown), which suggests that the Smuf2 level is controlled at the posttranscriptional level. Consistently, knockdown of PRMT1 reduced basal reporter activity and blocked TGF- $\beta$-mediated elevation of reporter activity. Although it is unclear whether or not elevation of Smurf2 level is due to lack of methylation of arginine residues, methylation of Smurf2 by PRMT1 may regulate stability of Smurf2 and control TGF- $\beta$ signaling.

\section{DISCUSSION}

We provide convincing evidence for the first time that Smurf2 is modified by PRMT1. Interestingly, Smurf1 is not methylated, although it has almost $80 \%$ sequence identity with Smurf2. Our results show that Smurf2 was methylated by PRMT1 in vitro and in vivo. In vitro methylation assay using PRMT1, PRMT3, PRMT4, and PRMT6 showed that only PRMT1 could catalyze Smurf2. Mapping of the methylation region using deletion constructs of Smurf2 showed that amino acid region 224-298 was methylated. Despite of lacking a GAR motif, there were two RXR motifs in the region. Substitution of putative methylated arginine residues to alanine caused reduced methylation, suggesting that these are novel methylation sites in Smurf2.

However, in the present study, we could not determine functional differences between wild-type Smurf2 and methylationdefective mutant Smurf2. Both wild-type and mutants were equally efficient in blocking TGF- $\beta$-mediated reporter activity, although this could be an artifact of the overexpression experiment, and did not show any difference in cellular localization (data not shown). The level of Smurf2 is regulated during the cell cycle (Osmundson et al., 2008). However, comparison of Smurf2 expression in wild-type and PRMT1 knockdown cells during cell cycle showed no significant difference (data not shown).

Interestingly, the level of Smurf2 increased upon knockdown of PRMT1. This result seems to be contradictory to the overexpression experiments shown in Fig. 5. Since we could not see any significant difference in the level of proteins when equal 
amount of plasmids for wild type or mutants on methylation sites were transfected (data not shown), it might be possible that PRMT1-mediated methylation at these sites is not involved in the regulation of Smurf2 level. Further study is necessary to confirm that elevation of Smurf2 expression is due to lack of methylation of arginine residues. Our data suggest that methylation of Smurf2 by PRMT1 may regulate stability of Smurf2 and control TGF- $\beta$ signaling. PRMT1 methylates Smad6 and allows phosphorylation of Smad1/5, which is necessary for transduction of BMP signaling (Xu et al., 2013). However, our report is the first case showing regulation of TGF- $\beta$ signaling by PRMT1. Previously, we showed that PRMT1 controls Wnt $/ \beta$-catenin signaling by methylation and stabilization of Axin (Cha et al., 2011). Current and previous data suggest that regulation of protein stability by PRMT1 is a key mechanism to control diverse signaling pathways.

\section{ACKNOWLEDGMENTS}

This research was supported by NRF grants funded by the Ministry of Science, ICT and Future Planning (2012R1A2A2A 01012472; 2012M3A9C6050109) to E. Jho.

\section{REFERENCES}

Bedford, M.T. (2007). Arginine methylation at a glance. J. Cell Sci. $120,4243-4246$.

Bedford, M.T., and Clarke, S.G. (2009). Protein arginine methylation in mammals: who, what, and why. Mol. Cell 33, 1-13.

Bedford, M.T., Frankel, A., Yaffe, M.B., Clarke, S., Leder, P., and Richard, S. (2000). Arginine methylation inhibits the binding of proline-rich ligands to Src homology 3 , but not WW, domains. J. Biol. Chem. 275, 16030-16036.

Blank, M., Tang, Y., Yamashita, M., Burkett, S.S., Cheng, S.Y., and Zhang, Y.E. (2012). A tumor suppressor function of Smurf2 associated with controlling chromatin landscape and genome stability through RNF20. Nat. Med. 18, 227-234.

Boisvert, F.M., Chenard, C.A., and Richard, S. (2005). Protein interfaces in signaling regulated by arginine methylation. Sci. STKE 2005, re2.

Cha, B., and Jho, E.H. (2012). Protein arginine methyltransferases (PRMTs) as therapeutic targets. Expert. Opin. Ther. Targets 16, 651-664.

Cha, B., Kim, W., Kim, Y.K., Hwang, B.N., Park, S.Y., Yoon, J.W., Park, W.S., Cho, J.W., Bedford, M.T., and Jho, E.H. (2011). Methylation by protein arginine methyltransferase 1 increases stability of Axin, a negative regulator of Wnt signaling. Oncogene 30, 2379-2389.

Deshaies, R.J., and Joazeiro, C.A. (2009). RING domain E3 ubiquitin ligases. Annu. Rev. Biochem. 78, 399-434.

Dupont, S., Inui, M., and Newfeld, S.J. (2012). Regulation of TGFbeta signal transduction by mono- and deubiquitylation of Smads. FEBS Lett. 586, 1913-1920.

Fukuchi, M., Fukai, Y., Masuda, N., Miyazaki, T., Nakajima, M., Sohda, M., Manda, R., Tsukada, K., Kato, H., and Kuwano, H. (2002). High-level expression of the Smad ubiquitin ligase Smurf2 correlates with poor prognosis in patients with esophageal squamous cell carcinoma. Cancer Res. 62, 7162-7165.

Goulet, I., Gauvin, G., Boisvenue, S., and Cote, J. (2007). Alternative splicing yields protein arginine methyltransferase 1 isoforms with distinct activity, substrate specificity, and sub- cellular localization. J. Biol. Chem. 282, 33009-33021.

Han, G., Li, A.G., Liang, Y.Y., Owens, P., He, W., Lu, S. Yoshimatsu, Y., Wang, D., Ten Dijke, P., Lin, X., et al. (2006). Smad7-induced beta-catenin degradation alters epidermal appendage development. Dev. Cell 11, 301-312.

Hershko, A., and Ciechanover, A. (1998). The ubiquitin system. Annu. Rev. Biochem. 67, 425-479.

Izzi, L., and Attisano, L. (2004). Regulation of the TGFbeta signalling pathway by ubiquitin-mediated degradation. Oncogene 23, 2071-2078

Jin, C., Yang, Y.A., Anver, M.R., Morris, N., Wang, X., and Zhang, Y.E. (2009). Smad ubiquitination regulatory factor 2 promotes metastasis of breast cancer cells by enhancing migration and invasiveness. Cancer Res. 69, 735-740.

Kim, S., and Jho, E.H. (2010). The protein stability of Axin, a negative regulator of Wnt signaling, is regulated by Smad ubiquitination regulatory factor 2 (Smurf2). J. Biol. Chem. 285, 36420-36426.

Lonn, P., Moren, A., Raja, E., Dahl, M., and Moustakas, A. (2009). Regulating the stability of TGFbeta receptors and Smads. Cel Res. 19, 21-35.

McBride, A.E., and Silver, P.A. (2001). State of the arg: protein methylation at arginine comes of age. Cell 106, 5-8.

McBride, A.E., Weiss, V.H., Kim, H.K., Hogle, J.M., and Silver, P.A. (2000). Analysis of the yeast arginine methyltransferase Hmt1p/Rmt1p and its in vivo function. Cofactor binding and substrate interactions. J. Biol. Chem. 275, 3128-3136.

Mukhopadhyay, D., and Riezman, H. (2007). Proteasomeindependent functions of ubiquitin in endocytosis and signaling. Science 315, 201-205.

Osmundson, E.C., Ray, D., Moore, F.E., Gao, Q., Thomsen, G.H., and Kiyokawa, H. (2008). The HECT E3 ligase Smurf2 is required for Mad2-dependent spindle assembly checkpoint. J. Cell Biol. 183, 267-277.

Pawlak, M.R., Scherer, C.A., Chen, J., Roshon, M.J., and Ruley, H.E. (2000). Arginine N-methyltransferase 1 is required for early postimplantation mouse development, but cells deficient in the enzyme are viable. Mol. Cell Biol. 20, 4859-4869.

Pickart, C.M. (2001). Mechanisms underlying ubiquitination. Annu Rev Biochem 70, 503-533.

Rotin, D., and Kumar, S. (2009). Physiological functions of the HECT family of ubiquitin ligases. Nat. Rev. Mol. Cell. Biol. 10, 398-409.

Tang, J., Frankel, A., Cook, R.J., Kim, S., Paik, W.K., Williams, K.R., Clarke, S., and Herschman, H.R. (2000). PRMT1 is the predominant type I protein arginine methyltransferase in mammalian cells. J. Biol. Chem. 275, 7723-7730.

Tiscornia, G., Singer, O., Ikawa, M., and Verma, I.M. (2003). A general method for gene knockdown in mice by using lentiviral vectors expressing small interfering RNA. Proc. Natl. Acad. Sci. USA 100, 1844-1848.

Xu, J., Wang, A.H., Oses-Prieto, J., Makhijani, K., Katsuno, Y., Pei, M., Yan, L., Zheng, Y.G., Burlingame, A., Bruckner, K., et al. (2013). Arginine Methylation Initiates BMP-Induced Smad Signaling. Mol. Cell 51, 5-19.

Yamagata, K., Daitoku, H., Takahashi, Y., Namiki, K., Hisatake, K., Kako, K., Mukai, H., Kasuya, Y., and Fukamizu, A. (2008). Arginine methylation of FOXO transcription factors inhibits their phosphorylation by Akt. Mol. Cell 32, 221-231.

Zhang, Y., Chang, C., Gehling, D.J., Hemmati-Brivanlou, A., and Derynck, R. (2001). Regulation of Smad degradation and activity by Smurf2, an E3 ubiquitin ligase. Proc. Natl. Acad. Sci. USA 98, 974-979. 